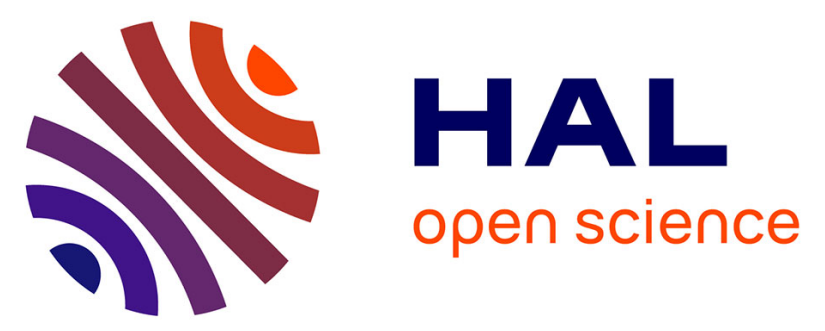

\title{
Cyclocoagulation of the ciliary bodies by high-intensity focused ultrasound: A 12-month multicenter study
} Philippe Denis, Florent Aptel, Jean-François Rouland, Jean-Philippe Nordmann, Yves Lachkar, Jean-Paul Renard, Eric Sellem, Christophe Baudouin, Alain Bron

\section{To cite this version:}

Philippe Denis, Florent Aptel, Jean-François Rouland, Jean-Philippe Nordmann, Yves Lachkar, et al.. Cyclocoagulation of the ciliary bodies by high-intensity focused ultrasound: A 12-month multicenter study. Investigative Ophthalmology \& Visual Science, 2015, 56 (2), pp.1089-1096. 10.1167/iovs.1414973 . hal-01220741

\section{HAL Id: hal-01220741 \\ https://hal.science/hal-01220741}

Submitted on 26 Oct 2015

HAL is a multi-disciplinary open access archive for the deposit and dissemination of scientific research documents, whether they are published or not. The documents may come from teaching and research institutions in France or abroad, or from public or private research centers.
L'archive ouverte pluridisciplinaire HAL, est destinée au dépôt et à la diffusion de documents scientifiques de niveau recherche, publiés ou non, émanant des établissements d'enseignement et de recherche français ou étrangers, des laboratoires publics ou privés. 


\title{
Cyclocoagulation of the Ciliary Bodies by High-Intensity Focused Ultrasound: A 12-Month Multicenter Study
}

\author{
Philippe Denis, ${ }^{1}$ Florent Aptel, ${ }^{2}$ Jean-François Rouland, ${ }^{3}$ Jean-Philippe Nordmann, ${ }^{4}$ \\ Yves Lachkar, ${ }^{5}$ Jean-Paul Renard, ${ }^{6}$ Eric Sellem, ${ }^{7}$ Christophe Baudouin, ${ }^{8}$ and Alain Bron ${ }^{9}$
}

${ }^{1}$ Hôpital de la Croix-Rousse, Service d'Ophtalmologie, Hospices Civils de Lyon, Lyon, France

${ }^{2}$ Service d'Ophtalmologie, Centre Hospitalier Universitaire de Grenoble, Grenoble, France

${ }^{3}$ Service d'Ophtalmologie, Hôpital Claude Huriez, CHRU de Lille, Lille, France

${ }^{4}$ Service d'Ophtalmologie 3, Centre Hospitalier National des Quinze-Vingts, Paris, France

${ }^{5}$ Institut du Glaucome, Fondation Hôpital Saint-Joseph, Paris, France

${ }^{6}$ Service d'Ophtalmologie, Hôpital d'Instruction des Armées du Val de Grâce, Paris, France

${ }^{7}$ Centre Ophtalmologique Kléber, Lyon, France

${ }^{8}$ Service d'Ophtalmologie 2, Centre Hospitalier National des Quinze-Vingts, Paris, France

${ }^{9}$ Service d'Ophtalmologie, Hôpital Général, Dijon, France

Correspondence: Philippe Denis, Department of Ophthalmology, Croix-Rousse University Hospital, 103, Grande Rue de la Croix-Rousse, 69317 Lyon, cédex 04, France; philippe.denis@chu-lyon.fr.

Submitted: June 14, 2014 Accepted: December 18, 2014

Citation: Denis P, Aptel F, Rouland J-F, et al. Cyclocoagulation of the ciliary bodies by high-intensity focused ultrasound: a 12-month multicenter study. Invest Ophthalmol Vis Sci. 2015;56:1089-1096. DOI:10.1167/ iovs.14-14973
Purpose. To evaluate the safety and efficacy of high-intensity focused ultrasound (HIFU) cyclocoagulation in reducing intraocular pressure (IOP) in patients with refractory glaucoma by using a novel miniaturized delivery device (EyeOP1).

Methods. We conducted a 12-month open-label multicenter prospective study (EyeMUST1 Study). Patients with primary (primary open-angle glaucoma [POAG]) or secondary refractory glaucoma were treated in two groups depending on the duration of each ultrasound shot (group 1: 4 seconds; group 2: 6 seconds). The primary efficacy outcome was based on IOP reduction at 6 and 12 months.

Results. Fifty-two patients were enrolled: 36 (69\%) had POAG and 16 (31\%) had secondary glaucoma. Group $1(n=24)$ and group $2(n=28)$ had similar demographics and baseline characteristics. In group 1, IOP was reduced from a mean preoperative value of $29.7 \pm 7.7$ $\mathrm{mm} \mathrm{Hg} \mathrm{(} n=3.5$ glaucoma medications) to a mean postoperative value of $21.3 \pm 6.7 \mathrm{~mm} \mathrm{Hg}$ $(n=3.5$ glaucoma medications) and $20.1 \pm 6.7 \mathrm{~mm} \mathrm{Hg}(n=3.2$ glaucoma medications) at 6 and 12 months, respectively. In group 2, IOP was reduced from a mean preoperative value of $29.0 \pm 7.4 \mathrm{~mm} \mathrm{Hg}(n=3.3$ glaucoma medications) to a mean postoperative value of $20.2 \pm$ $7.4 \mathrm{~mm} \mathrm{Hg}(n=3.4$ glaucoma medications) and $18.5 \pm 6.6 \mathrm{~mm} \mathrm{Hg}(n=3.5$ glaucoma medications) at 6 and 12 months, respectively. At 12 months, the IOP reduction was sustained in both groups (32\% IOP reduction in group 1 and 36\% IOP reduction in group 2). The overall tolerance of the technique was good, with no serious adverse events.

Conclusions. The new miniaturized HIFU EyeOP1 delivery device seems to be effective in decreasing IOP in patients with refractory glaucoma. The technology offers a good safety profile. (ClinicalTrials.gov number, NCT01338467.)

Keywords: ciliary body/ultrasonography, glaucoma/therapy, intraocular pressure, miniaturization/methods, treatment outcome
Tntraocular pressure (IOP) reduction is the only glaucoma therapy proven to be effective. It has been shown to delay or prevent the development of glaucoma in eyes with ocular hypertension $^{1}$ and to prevent progression of glaucoma in eyes with and without elevated IOP. ${ }^{2,3}$ Intraocular pressure reduction can be achieved by topical and systemic medications, various laser therapies, and a number of incisional surgical techniques. These approaches aim to reduce IOP through a better balance between aqueous humor inflow and outflow: increasing outflow and/or decreasing inflow, that is, aqueous humor production.

Diode laser cyclophotocoagulation (CPC) is considered to be the current ciliary body ablation technique to lower IOP. ${ }^{4}$ Historical methods, such as diathermy and cryotherapy, have been used with poor visual status and are associated with serious and vision-impairing complications. ${ }^{4,5}$

Laser diode cycloablation has been shown to be reasonably effective but may cause destruction of surrounding ocular tissues, with a significant risk of chronic ocular hypotony, phthisis, uveal inflammation, and retinal detachment. ${ }^{4-10}$

To selectively destroy ciliary body tissue, high-intensity focused ultrasound (HIFU) was introduced first in the United States by Coleman et al. ${ }^{11}$ (Sonocare Therapeutic Ultrasound System; Sonocare, Inc., Ridgewood, NJ, USA) and later in Europe. Positive outcomes decreasing IOP have been reported. ${ }^{11-13}$ Compared to lasers, ultrasound beams can be focused through optically opaque ocular media with controlled energy absorption, thereby minimizing the impact upon adjacent structures, and their effect does not depend on the degree of 
pigmentation of the ciliary body. Despite being effective in reducing IOP, the former HIFU procedure is burdensome, requiring up to 2 hours in the operating $\operatorname{room}^{11,14}$ and is technically challenging owing to a rather bulky delivery system and the complex management of multiple confluent impacts. Moreover, the procedure is associated with a relatively high rate of ocular complications, probably due to the inaccurate positioning of the bulky probe. Furthermore, the relatively low frequency of the device $(5 \mathrm{MHz})$ creates a much wider focal zone, thus potentially damaging more tissue than necessary. ${ }^{15,16}$

Leveraging recent advances in the technology, a new cyclocoagulation device, the EyeOP1 (EyeTechCare, Rillieuxla-Pape, France), using miniaturized transducers to produce HIFU, has been developed. The device has previously been described in detail. ${ }^{16-18}$ Several technical improvements have been made to the previous ultrasound technology, providing simpler, safer, faster, and more precise treatment. More specifically, the therapy probe is placed in direct contact with the ocular surface. The entire treatment is conducted with no need to modify the settings, which significantly reduces the procedure time (approximately 2 minutes) and minimizes the risk of operator errors. The rapid sequential activation of the miniaturized transducers delivers six focused ultrasound beams to induce partial and well-controlled lesions corresponding to six segments of linear tissue coagulation in the ciliary body (cyclocoagulation). The higher operating frequency $(21 \mathrm{MHz})$ than that of the Sonocare system $(5 \mathrm{MHz})$ allows for a sharper transition between the focal zone and adjacent untreated tissue. The result is a highly precise focusing of the HIFU target zone, not exceeding $0.1 \mathrm{~mm} \times 1 \mathrm{~mm}$, enhanced by preoperative high-resolution modeling of the ocular structures. The treatment parameters include a $21-\mathrm{MHz}$ frequency, $2.45-\mathrm{W}$ acoustic power, with the activation of each transducer lasting 4 or 6 seconds, depending on patient groups. The HIFU probe is supplied in three sizes $(11,12$, and $13 \mathrm{~mm})$, which fit most ocular sizes and, for every patient, the choice of the right size is based directly on ultrasound biomicroscopy (UBM) preoperative biometric data. ${ }^{17}$

Animal studies using the EyeOP1 device have shown a reduction in IOP with good local tolerance, confirmed by histologic examination. ${ }^{18}$ Subsequently, a pilot clinical study in 12 refractory glaucoma patients was conducted, showing a significant IOP reduction with no major intra- or postoperative complications, using 3- and 4-second treatment times. ${ }^{17}$ Based on the results of the pilot study, a new dose-escalation study was conducted with 4 - and 6-second treatment times, mainly to compare the efficacy results and safety profile with the two exposure times.

\section{Patients and Methods}

Fifty-two patients with primary and secondary glaucoma of various etiologies (Table 1), including primary open-angle glaucoma (POAG) with a history of previous failed filtering surgery, pigmentary glaucoma, and pseudo-exfoliation glaucoma, were recruited from nine French clinical sites under a prospective open-label study of the EyeOP1 device (Table 1). The EyeMUST1 study was registered on ClinicalTrials.gov under the identifying number NCT01338467. The study was conducted in compliance with the Declaration of Helsinki, ISO 14155:2011: Clinical Investigations of Medical Devices for Human Subjects-Good Clinical Practices, and after approval from relevant ethics committees and the competent authorities. All patients provided written informed consent before enrollment. Eligibility criteria included men or women 18 years or older, IOP $>21 \mathrm{~mm} \mathrm{Hg}$ under maximum medical therapy,
Table 1. Patient Demographics

\begin{tabular}{|c|c|c|c|}
\hline$N(\%)$ & Group 1 & Group 2 & $P$ Value \\
\hline Patients & 24 & 28 & \\
\hline Age, mean (range), y & $63.8(40-89)$ & $62.5(37-88)$ & NS \\
\hline Sex & & & $1.00^{*}$ \\
\hline Female & $12(50.0)$ & $14(50.0)$ & \\
\hline Male & $12(50.0)$ & $14(50.0)$ & \\
\hline Ethnicity & & & $0.123 \dagger$ \\
\hline Caucasian & $18(75.0)$ & $26(92.8)$ & \\
\hline Black & $6(25.0)$ & $2(7.2)$ & \\
\hline Type of glaucoma & & & $0.313 \dagger$ \\
\hline Primary open-angle & $14(58.3)$ & $22(78.6)$ & \\
\hline Uveitic & $3(12.5)$ & 0 & \\
\hline Exfoliative & $1(4.2)$ & $2(7.1)$ & \\
\hline Pigmentary & $1(4.2)$ & 0 & \\
\hline Traumatic & $1(4.2)$ & 0 & \\
\hline Aphakic & $1(4.2)$ & $1(3.6)$ & \\
\hline Others & $3(12.5)$ & $3(10.7)$ & \\
\hline Juvenile & 2 & 0 & \\
\hline Chandler syndrome & 1 & 0 & \\
\hline Ocular toxoplasmosis & 0 & 1 & \\
\hline Angle-closure glaucoma & 0 & 2 & \\
\hline
\end{tabular}

with at least one failed filtering surgery. Patients included in the study had not had surgical or laser treatment within the 3 months preceding the study treatment, and no previous ciliary body interventions or drainage implants were included. Additionally, prior hypotensive treatments were maintained throughout the study period. Only one eye per patient was eligible for enrollment.

Two consecutive groups of patients treated with two different exposure times were included. The patients in group 1 were treated with a 4-second insonification exposure time and the patients in group 2 were treated with a 6-second exposure time.

The 4-second exposure time was the dose used in the initial pilot study, showing an acceptable compromise in terms of efficacy and safety. To confirm the results of the pilot study on a broader population with a greater number of centers involved, this study started at the 4-second exposure time.

The dose-escalation design was planned to allow the HIFU duration to be increased from 4 to 6 seconds after a 1-month interim review of the results of the first pool of patients by the investigator committee. Based on preclinical studies, increasing the HIFU delivery time from 4 to 6 seconds was intended to increase the volume of ciliary process destruction from 4.8 $\mathrm{mm}^{3}$ to $7.8 \mathrm{~mm}^{3}$ each, and therefore to increase the overall effect on inflow reduction. ${ }^{15,16}$

The study lasted 1 year, with the primary effectiveness endpoint evaluated at 6 and 12 months. Postoperative visits occurred at 1 day, 1 week, and then 1, 2, 3, 6, and 12 months. The ocular examination included slit-lamp and fundus examination, Snellen best corrected visual acuity (BCVA), central visual field, ultrasonic corneal pachymetry, gonioscopy, preoperative axial length measurement, UBM, and optical coherence tomography (AC Visante OCT; Carl Zeiss Meditec AG, Jena, Germany) examinations. Intraocular pressure, measured with Goldman applanation tonometry, was requested to be taken within \pm 3 hours of the preoperative examination time. The use of hypotensive medication and any adverse events were recorded at each visit. As per protocol, the glaucoma medication remained unchanged for the 2 months following 

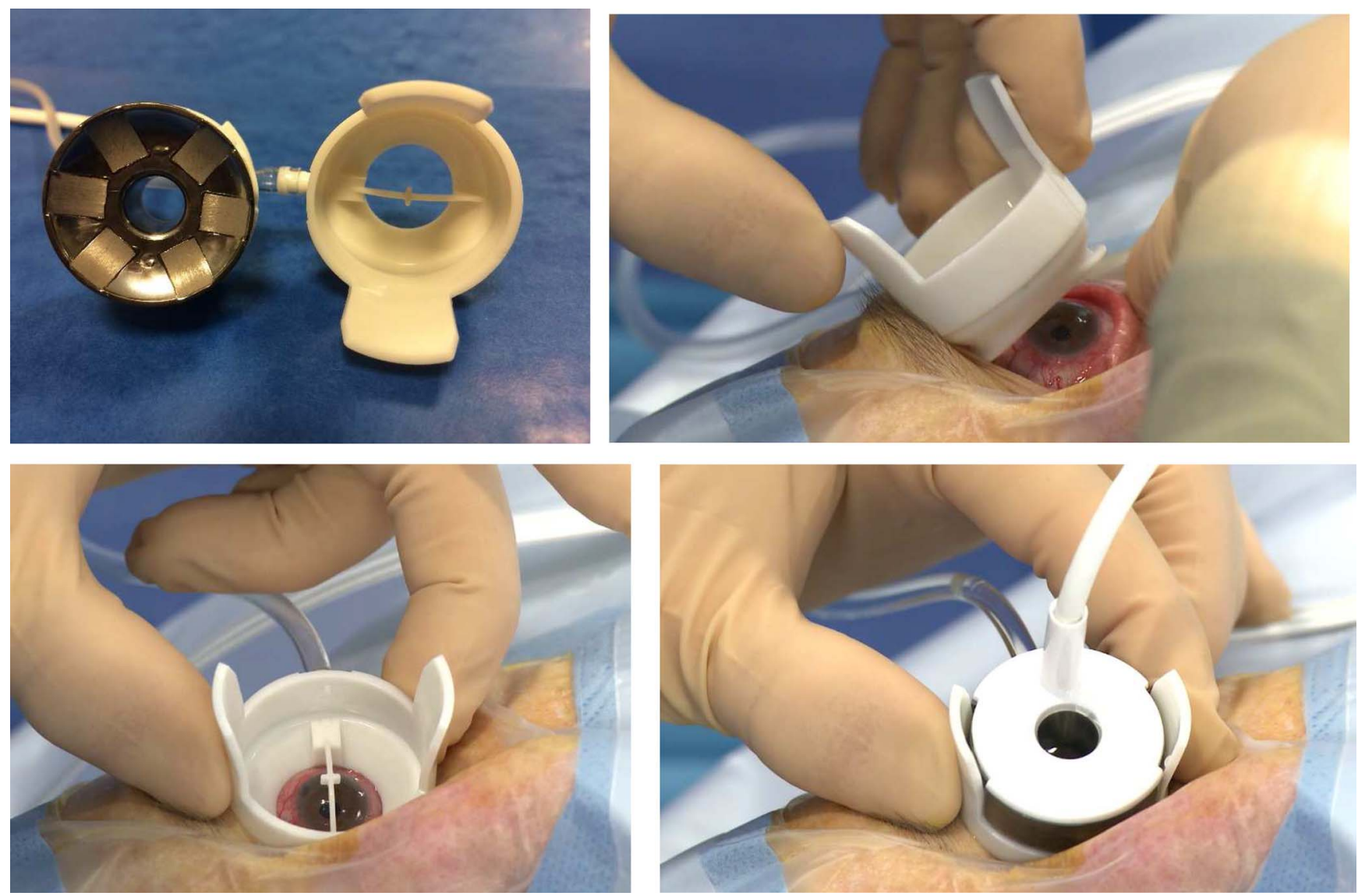

FiguRE 1. Top left: High-intensity focused ultrasound device comprising two elements: the probe (left) with the six piezoelectric elements (transducers) generating the ultrasound beam, and the positioning cone (right). Bottom left: The cone in place showing a ring of visible sclera; when this ring is regular, the position is correct and then maintained by a mild vacuum system. Bottom right: The probe has been inserted in the cone and the device has been filled with physiological solution via the well in the middle. The treatment can start.

the study procedure. After the 2-month period, if necessary, an adjustment of the patient's medication was allowed. Also, after 2 months, retreatment was allowed for patients with either IOP remaining above $28 \mathrm{~mm} \mathrm{Hg}$ despite initial treatment efficacy, that is, a $>20 \%$ reduction, or for patients who did not experience a $20 \%$ decrease versus preoperative values.

\section{HIFU Procedure}

Patients were treated under peribulbar anesthesia, general anesthesia, or topical anesthesia combined with short sedation, according to each center's specific preferences. All the investigators were carefully trained in the EyeOP1 procedure (Fig. 1). To focus the ablation in the ciliary body, the HIFU probe size was selected on the basis of anatomy of each eye, assessed in all patients by UBM ultrasonography of the anterior segment. Moreover, in several equipped centers, a Visante ASOCT examination was done (25/54 patients), but the OCT results were not used to determine probe size. All probe sizes were selected in the study management center by a single operator using a proprietary computer-assisted overlay drawing method performed by the EyeOP1 manufacturer. Three device models with different ring diameters, equipped with the six transducers, were available. Depending on the diameter, the six elliptical cylinder-shaped volumes were centered on an 11-, 12-, or 13-mm-diameter circle. After sizing, the probe was manually centered on the patient's eye and held in place by a mild suction system during the sequential activation of the six sectors.
In addition to the pressure-lowering preoperative treatment requested to be maintained for the first 2 months after the HIFU procedure, postoperative treatments as per protocol included flurbiprofen (Ocufen; Allergan, Irvine, CA, USA) or a combination of dexamethasone and tobramycin (Tobradex; Alcon Laboratories, Inc., Fort Worth, TX, USA) given four times daily for 1 month.

\section{Outcome Measures and Statistical Analysis}

The primary efficacy outcome was based on IOP reduction at 6 and 12 months. Surgical success (patients considered to be responders) was IOP reduction from baseline $\geq 20 \%$ and final IOP $>5 \mathrm{~mm} \mathrm{Hg}$ without adding hypotensive medications and with possible HIFU retreatment.

Secondary endpoints were intra- and postoperative tolerance and visual acuity. Other study outcomes included IOP and medication use results, and complication rates.

The key outcome measures are presented by using descriptive statistics. The Student's $t$-test was used to compare means and percentages; statistical significance was set at $P<$ 0.05. Owing to the descriptive nature of the statistical analysis, no imputations of missing data were performed, and the analyses used only observed data.

The study protocol design stated that in case an additional treatment, such as filtering surgery or cyclodestruction (CPC laser or cryotherapy), was administered to a patient to lower ocular pressure, the patient would be considered as having failed the HIFU treatment (failure). Efficacy data (IOP values) 


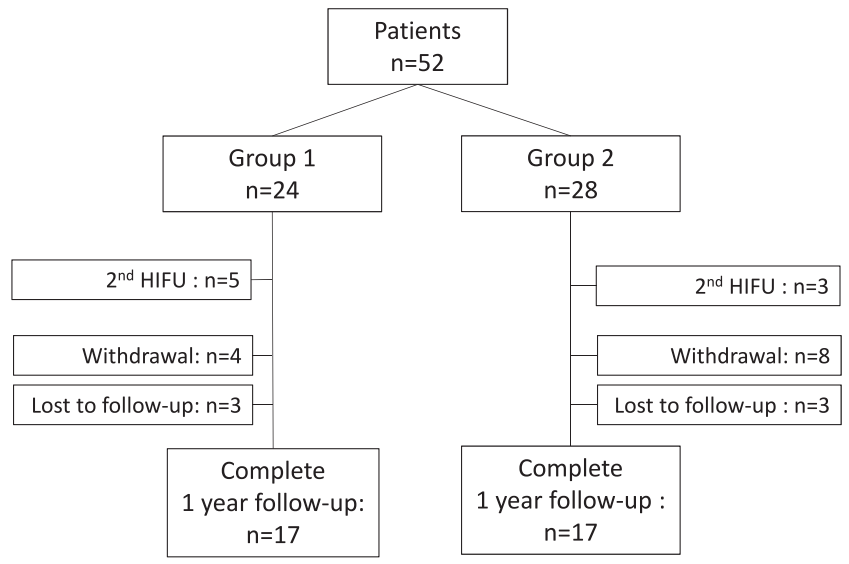

Figure 2. Flow chart.

would not be collected and integrated into the results for patients withdrawn from the study after undergoing filtering surgery or CPC treatment by diode laser so as not to bias the results.

\section{Results}

\section{Demographics}

Fifty-two (52) patients were enrolled in the study (Fig. 2): 24 were treated with the 4-second dose (group 1) and 28 with the 6-second dose (group 2). Demographic data are reported in Table 1. The HIFU procedure was incomplete for two patients: one experienced chemosis, impairing the ability to deliver the planned treatment, and for another patient, only four of the six planned sectors were treated. Nevertheless, these two patients were included in the efficacy and safety analysis. The enrollment rate between the two groups was overall evenly distributed across the sites. Only one eye per patient was treated.

Table 2 presents the ocular characteristics of the population before the study treatment, per study group.

All HIFU procedures were performed by 10 surgeons, under local anesthesia $(n=38)$, topical with sedation $(n=7)$, or general $(n=7)$ anesthesia depending on patient and physician preference. Forty-four patients had only one HIFU procedure and eight patients had two HIFU procedures.

\section{Efficacy in All Patients}

The efficacy results include the patients who were re-treated as planned as per protocol for five (5/24) patients in group 1 and three $(3 / 28)$ patients in group 2 .

The effect of the HIFU procedure on IOP was clinically significant for both groups (Table 3 ).

At 6 months, IOP was significantly reduced in group 1 from $29.7 \mathrm{~mm} \mathrm{Hg}$ (SD, $7.7 \mathrm{~mm} \mathrm{Hg}$ ) to $21.3 \mathrm{~mm} \mathrm{Hg}$ (SD, $6.7 \mathrm{~mm} \mathrm{Hg}$ ), corresponding to a mean reduction of $28.3 \%$; and in group 2 from $29.0 \mathrm{~mm} \mathrm{Hg}$ (SD, $7.4 \mathrm{~mm} \mathrm{Hg}$ ) to $20.2 \mathrm{~mm} \mathrm{Hg}$ (SD, $7.4 \mathrm{~mm}$ $\mathrm{Hg}$ ), corresponding to a mean IOP reduction of $30.2 \%$. These results were obtained with virtually no change in glaucoma medication in either group.

At 12 months, IOP reduction was sustained in both groups with a mean IOP of $20.1 \mathrm{~mm} \mathrm{Hg}(\mathrm{SD}, 6.7 \mathrm{~mm} \mathrm{Hg}$ ) in group 1 (corresponding to a mean reduction of $32.2 \%$ ) and a mean IOP of $18.5 \mathrm{~mm} \mathrm{Hg}(\mathrm{SD}, 6.6 \mathrm{~mm} \mathrm{Hg}$ ) in group 2 (corresponding to a mean reduction of $36.0 \%$ ) (Fig. 3).

Success, defined as a greater than $20 \%$ IOP decrease and IOP $>5 \mathrm{~mm} \mathrm{Hg}$ was achieved at 6 months in $61.9 \%(13 / 21)$ of the patients in group 1 and $65.4 \%(17 / 26)$ of the patients in group 2. At 12 months, this proportion was $57.1 \%$ in group 1 $(12 / 21)$ and $48.0 \%$ in group $2(12 / 25)$. This difference was not statistically significant (Fisher test, $P$ value $=0.56$ ) .

\section{Efficacy in POAG Patients}

The success rate of the POAG subgroups significantly differed from that observed in the overall population (Table 4).

At 6 months, IOP was significantly reduced in group 1 POAG patients from $28.0 \mathrm{~mm} \mathrm{Hg}$ (SD, $5.0 \mathrm{~mm} \mathrm{Hg}$ ) to $20.3 \mathrm{~mm}$ $\mathrm{Hg}$ (SD, $5.7 \mathrm{~mm} \mathrm{Hg}$ ), corresponding to a mean IOP reduction of $27.5 \%$; and in group 2 POAG patients, from $28.7 \mathrm{~mm} \mathrm{Hg}$ (SD, $6.8 \mathrm{~mm} \mathrm{Hg}$ ) to $18.8 \mathrm{~mm} \mathrm{Hg}(\mathrm{SD}, 5.1 \mathrm{~mm} \mathrm{Hg}$ ), corresponding to a mean IOP reduction of $34.5 \%$. These results were obtained with virtually no change in glaucoma medications in either group.

At 12 months, IOP reduction was sustained in both groups with a mean IOP of $19.0 \mathrm{~mm} \mathrm{Hg}(\mathrm{SD}, 5.6 \mathrm{~mm} \mathrm{Hg})$ in group 1

TABLE 2. Patient Ocular Characteristics

\begin{tabular}{|c|c|c|c|}
\hline$N(\%)$ & Group 1, $n=24$ & Group $2, n=28$ & $P$ Value \\
\hline No. of previous filtering glaucoma surgeries & & & $0.466^{*}$ \\
\hline$n=1$ & $15(62.5)$ & $17(60.7)$ & \\
\hline$n=2$ & $6(25.0)$ & $10(35.7)$ & \\
\hline$n \geq 3$ & $3(12.5)$ & $1(3.6)$ & \\
\hline Lens status & & & $0.887^{*}$ \\
\hline Phakic & $11(45.8)$ & $15(53.6)$ & \\
\hline Pseudophakic & $12(50.0)$ & $12(42.8)$ & \\
\hline Aphakic & $1(4.2)$ & $1(3.6)$ & \\
\hline \multicolumn{4}{|l|}{ Preoperative mean values (range) } \\
\hline $\mathrm{IOP}$, mean $\pm \mathrm{SD}, \mathrm{mm} \mathrm{Hg}$ & $29.7 \pm 7.7$ & $29.0 \pm 7.4$ & $0.739 \dagger$ \\
\hline No. of glaucoma medications, eyedrops & $2.9(1-4)$ & $2.8(0-4)$ & $0.776 \dagger$ \\
\hline No. of patients with preoperative systemic carbonic anhydrase inhibitors & $7(29.1)$ & $7(25.0)$ & $0.735 \ddagger$ \\
\hline No. of previous glaucoma surgeries & $1.54(1-4)$ & $1.50(1-4)$ & $0.847 \dagger$ \\
\hline BCVA, logMAR & 0.98 & 0.94 & $0.899 \dagger$ \\
\hline
\end{tabular}

\footnotetext{
${ }^{*}$ Fisher test.

† Student's $t$-test.

$\ddagger \chi^{2}$ test.
} 

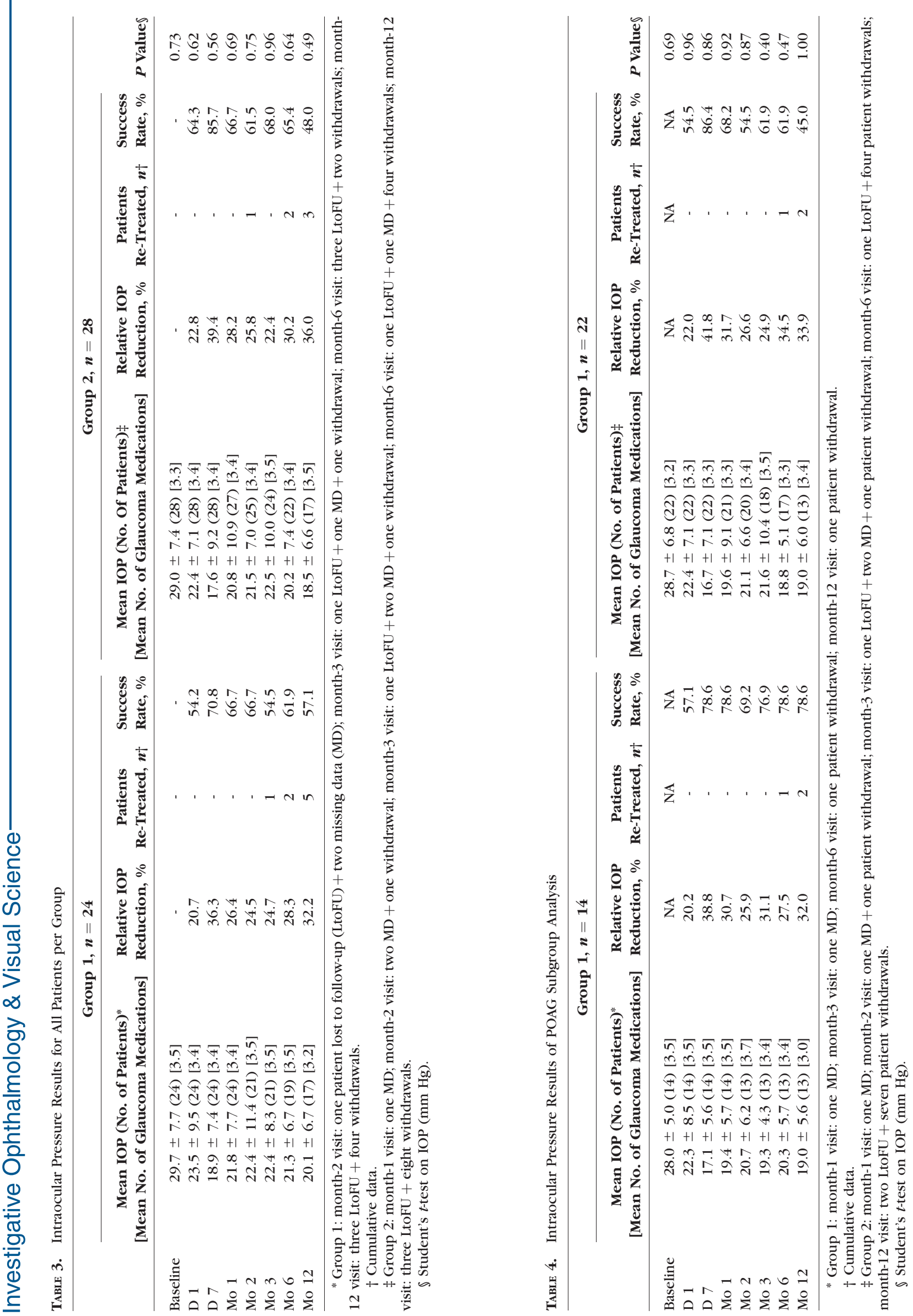
TABLE 5. Intra- and Postoperative Ocular Complications

\begin{tabular}{lcc}
\multicolumn{1}{c}{ Ocular Complications } & Group 1 & Group 2 \\
\hline Intraoperative & & \\
Pain & 1 & 3 \\
Corneal burn & 0 & 0 \\
Subconjunctival hemorrhage & 1 & 1 \\
Postoperative & & \\
Hyperemia & 9 & 16 \\
Superficial punctuated keratitis & 6 & 11 \\
Corneal edema & 2 & 2 \\
Ocular pain & 1 & 2 \\
Anterior chamber reaction & 6 & 7 \\
Transient hypotonia, choroidal detachment & 0 & 1 \\
Transient macular edema & 0 & 1 \\
Phthisis & 0 & 0 \\
Cataract & 0 & 0 \\
Intravitreous hemorrhage & 0 & 0 \\
Loss of visual acuity ( $>2$ lines) & $3(12.5 \%)$ & $3(10.7 \%)$ \\
\end{tabular}

POAG patients (32.0\% IOP reduction) and a mean IOP of 19.0 $\mathrm{mm} \mathrm{Hg}$ (SD, $6 \mathrm{~mm} \mathrm{Hg}$ ) in group 2 POAG patients (33.9\% IOP reduction).

Success, defined as an IOP decrease greater than $20 \%$ and IOP $>5 \mathrm{~mm} \mathrm{Hg}$ was achieved at 6 months in $78.6 \%(11 / 14)$ of group 1 POAG patients and 61.9\% (13/21) of group 2 POAG patients. This difference was not statistically significant (Fisher test, $P$ value $=0.46$ ).

At 12 months, this proportion was $78.6 \%$ in group 1 POAG patients (11/14) and $45.0 \%$ in group 2 POAG patients $(9 / 20)$. This difference was not statistically significant (Fisher test, $P$ value $=0.079$ ).

\section{Tolerance}

Safety information was reported for 52 subjects, including the two patients for whom the treatment was not administered as per protocol.

\section{Intraoperative Complications}

Few minor intraoperative complications were reported. As mentioned above, 38 patients received the procedure under peribulbar anesthesia, 7 under topical anesthesia, and 7 under general anesthesia. A total of 4 of 52 patients reported tolerable pain during the procedure (one patient in group 1 and three patients in group 2). In all cases, intraoperative pain was transient and disappeared immediately at the end of the procedure. Two subjects had intraoperative subconjunctival hemorrhage.

Group 1

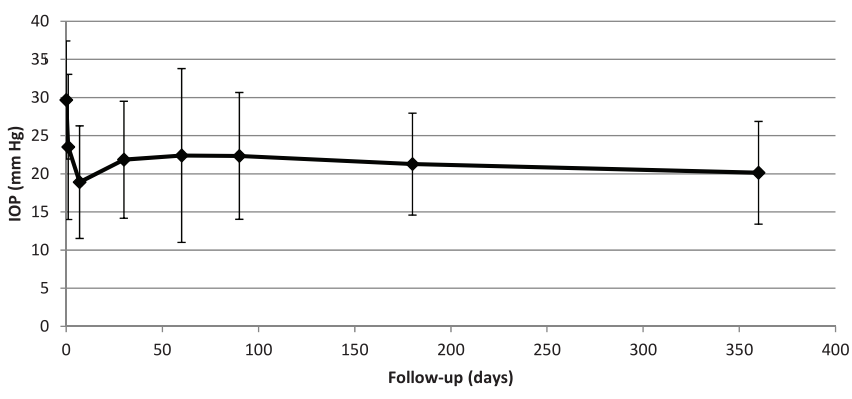

FigURE 3. Mean IOP per group for the 12 months after HIFU treatment.
One patient in group 1 presented an IOP spike of $8 \mathrm{~mm} \mathrm{Hg}$ above baseline, which resolved within 1 hour of instillation of apraclonidine hydrochloride (Iopidine 0.5\%; Alcon Laboratories).

No corneal burns were observed during the procedure.

\section{Postoperative Complications}

The postoperative complications are listed in Table 5 . Seventeen cases (33\%) of superficial punctate keratitis resolved spontaneously in a few days and two cases of blepharitis were also reported. Other minor events reported included conjunctival hyperemia for 25 patients (48\%), 9 in group 1 and 16 in group 2; transient anterior chamber uveal reaction in 13 patients, 6 in group 1 and 7 in group 2; and transient corneal edema for 4 patients $(7.7 \%), 2$ in group 1 and 2 in group 2 .

Only one patient belonging to group 2 presented transitory postoperative hypotony (IOP $=4 \mathrm{~mm} \mathrm{Hg}$ ), associated with choroidal detachment. Antiglaucoma drugs were tapered off, additional topical steroid treatment was given, and the event resolved within 30 days.

Lastly, 12 patients required a secondary glaucoma surgical intervention for insufficient response of HIFU treatment, 9 with trabeculectomy, 2 with diode laser cyclodestruction, and 1 with Ahmed valve surgery. These treatments occurred between 6 and 12 months after the HIFU procedure. The study protocol design stated that if an additional treatment such as filtering surgery or cyclodestruction (CPC laser or cryotherapy) was administered, the patient would be considered as having failed the HIFU treatment (failure) and would be withdrawn from the study. Thus, efficacy data (IOP values) were not collected and integrated into the results for the patients withdrawn from the study after undergoing filtering surgery or CPC treatment by diode laser so as not to bias the results. This treatment was performed in 12 patients before the protocol visit planned twelve months after the procedure (four patients in group 1 and eight patients in group 2).

There was one report of transient macular edema in a group 2 patient, appearing 1 month after the HIFU procedure, resolving after 1 month under topical nonsteroidal antiinflammatory agents with no impact on visual acuity. There was no report of treatment-induced cataract.

\section{Visual Outcomes}

Mean visual acuity remained statistically unchanged (BCVA logMAR of $0.98 \pm 1.20$ and $1.09 \pm 1.18$ before and at last follow-up in group 1 , respectively; and $0.94 \pm 1.18$ and $1.24 \pm$ 1.36 before and at last follow-up in group 2 , respectively).

Six patients presented with a loss of BCVA of more than two lines: three in group $1(3 / 24,12.5 \%)$ and three in group $2(3 /$ $28,10.7 \%)$.

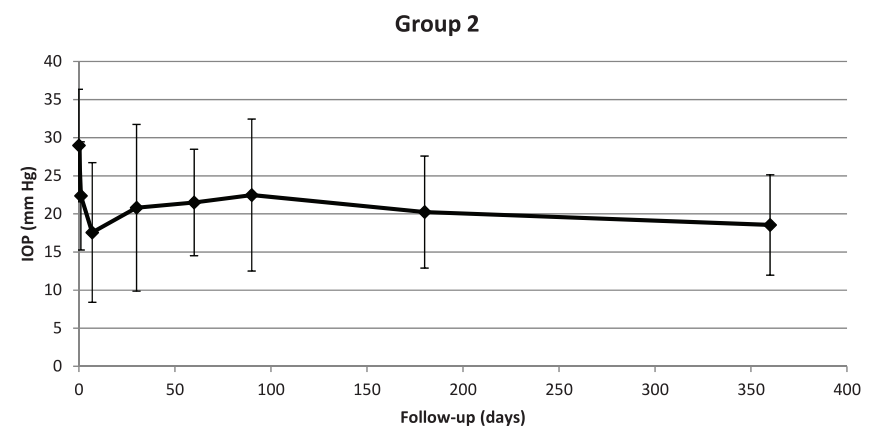



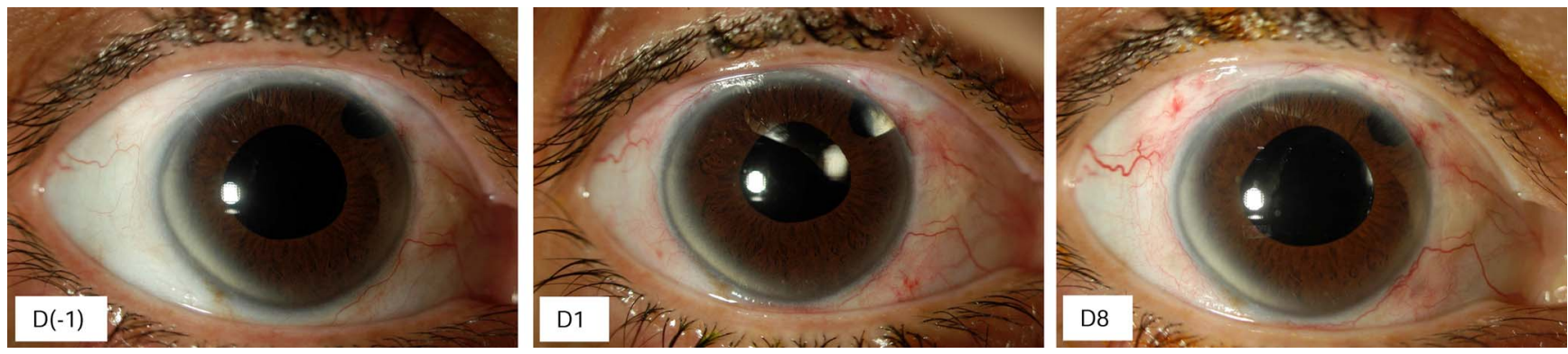

FiguRE 4. Ocular tolerance: Slit-lamp photographs taken preoperatively on the day before (left), 1 day after (middle), and 8 days after the procedure (right) (4-second HIFU treatment)

In group 1 , loss of BCVA in two patients was deemed unrelated to the study treatment (one case of cataract worsening after vitrectomy for retinal detachment and one case of central venous occlusion at 12 months).

In group 2, one patient who experienced a loss of BCVA underwent corneal decompensation of his corneal graft and one patient presented reactivation of toxoplasmosis choroiditis 1 month after the procedure.

\section{DisCuSSION}

Two recent previous studies ${ }^{17,19}$ have been conducted with this new device for ultrasound circular cyclocoagulation. The initial pilot study examined patients with refractory primary or secondary glaucoma (at least one previous incisional glaucoma surgery) and limited residual visual acuity and visual field (BCVA $<20 / 60$; visual field defect located in the paracentral region). ${ }^{17}$ The patients included have POAG, neovascular glaucoma, congenital glaucoma, primary angle-closure glauco$\mathrm{ma}$, and iridocorneal endothelial glaucoma. Intraocular pressure is reduced from a mean preoperative value of $37.9 \pm 10.7$ $\mathrm{mm} \mathrm{Hg}$ to a mean postoperative value of $26.3 \pm 5.1 \mathrm{~mm} \mathrm{Hg}$ at 6 months and $24.7 \pm 8.5 \mathrm{~mm} \mathrm{Hg}$ at the last follow-up visit. A $33.9 \%$ IOP reduction has been obtained at the last follow-up visit. Surgical success (defined by IOP reduction $\geq 20 \%$ and IOP $>5 \mathrm{~mm} \mathrm{Hg}$ ) has been obtained in 10 of 12 patients $(83.3 \%)$ at the last visit. The second study was conducted in 28 patients with POAG and much less advanced disease. ${ }^{19}$ Intraocular pressure is reduced from a mean preoperative value of $29.0 \pm 7.2 \mathrm{~mm} \mathrm{Hg}$ to a mean value of $21.6 \pm 9.4 \mathrm{~mm}$ $\mathrm{Hg}$ at last follow-up ( $n=1.29$ procedures) (mean IOP reduction, 26\%). Complete success (IOP reduction $>20 \%$ without reintervention and without additional hypotensive medications) has been achieved in $50 \%$ of eyes at the last follow-up (mean IOP reduction $45 \%$ in these eyes) and qualified success (IOP reduction $>20 \%$ and IOP $>5 \mathrm{~mm} \mathrm{Hg}$ with possible reinterventions) has been achieved in $68 \%$ of eyes at the last follow-up.

The present study was designed and conducted to evaluate the efficacy and safety of ultrasonic circular cyclocoagulation in patients with both primary and secondary glaucoma and with two different exposure times in a larger multicenter clinical trial.

Fifty-two patients with POAG or secondary glaucoma were enrolled and followed up during at least 12 months. In the 4second exposure time group, IOP was reduced from a mean preoperative value of $29.7 \pm 7.7 \mathrm{~mm} \mathrm{Hg}$ to a mean value of $20.1 \pm 6.7 \mathrm{~mm} \mathrm{Hg}$ and success (IOP reduction $>20 \%$ and IOP $>5 \mathrm{~mm} \mathrm{Hg}$ with possible retreatment) was achieved in $57.1 \%$ of eyes at 1 year of follow-up. In the subgroup of POAG patients, IOP was reduced from a mean preoperative value of $28.0 \pm 5.0 \mathrm{~mm} \mathrm{Hg}$ to a mean value of $19.0 \pm 5.6 \mathrm{~mm} \mathrm{Hg}$ and success was achieved in $78.6 \%$ of eyes at 1 year of follow-up. In the 6-second exposure time group, IOP was reduced from a mean preoperative value of $29.0 \pm 7.4 \mathrm{~mm} \mathrm{Hg}$ to a mean value of $18.5 \pm 6.6 \mathrm{~mm} \mathrm{Hg}$ and success (IOP reduction $>20 \%$ and IOP $>5 \mathrm{~mm} \mathrm{Hg}$ with possible retreatment) was achieved in $48 \%$ of eyes at 1 year of follow-up. In the subgroup of patients with POAG, IOP was reduced from a mean preoperative value of $28.7 \pm 6.8 \mathrm{~mm} \mathrm{Hg}$ to a mean value of $19.0 \pm 6.0 \mathrm{~mm} \mathrm{Hg}$ and success was achieved in $45 \%$ of eyes at 1 year of follow-up.

We found lower efficacy in patients with secondary glaucoma. One explanation could be that the amount of ciliary body tissue coagulated and the related decrease in aqueous humor production was sometimes insufficient, particularly in patients with secondary glaucoma. Since patients with secondary glaucoma could have a lower trabecular meshwork outflow of aqueous humor, a similar decrease in aqueous production could have a lesser effect on IOP. Some patients with an IOP response insufficient to reach the target IOP were re-treated. The six transducers were activated and the probe was rotated with the intention of targeting different areas of the ciliary body. The diameter of the probe was generally changed for the second treatment ( $n=3 / 8$ eyes re-treated without diameter change, 4/8 increased, 1/8 decreased). Intraocular pressure decreased significantly for three eyes after retreatment. This finding could corroborate the hypothesis that the amount of ciliary body tissue treated during the first procedure may be insufficient.

Animal studies conducted with the device have shown circumferentially distributed coagulation necrosis of the ciliary processes and ciliary body, particularly with a complete loss of the ciliary epithelium, likely resulting in reduced aqueous production. However, UBM examinations performed before and after the treatment in the first clinical studies have also shown hyporeflective suprachoroidal fluid spaces in patients having significantly lower IOP than those without visible suprachoroidal space, suggesting that ultrasound cyclocoagulation could also increase the uveoscleral outflow through the supraciliary and suprachoroidal space. The amount of ciliary body tissue destroyed, and therefore probably the reduction of aqueous production, increases with increasing dose. In contrast, one possibility would be that the relationship between the dose and the effect on the uveoscleral pathway may not be linear. We can hypothesize that exposure to a small dose of ultrasound energy leads to tissue retraction or tissue microarchitecture changes increasing the permeability to aqueous humor, whereas greater exposure to ultrasound energy coagulates the uveoscleral tract and decreases the related aqueous outflow. A more definitive answer would require in vivo fluorophotometric and tonographic quantification of the aqueous inflow and outflow with various exposure times. 


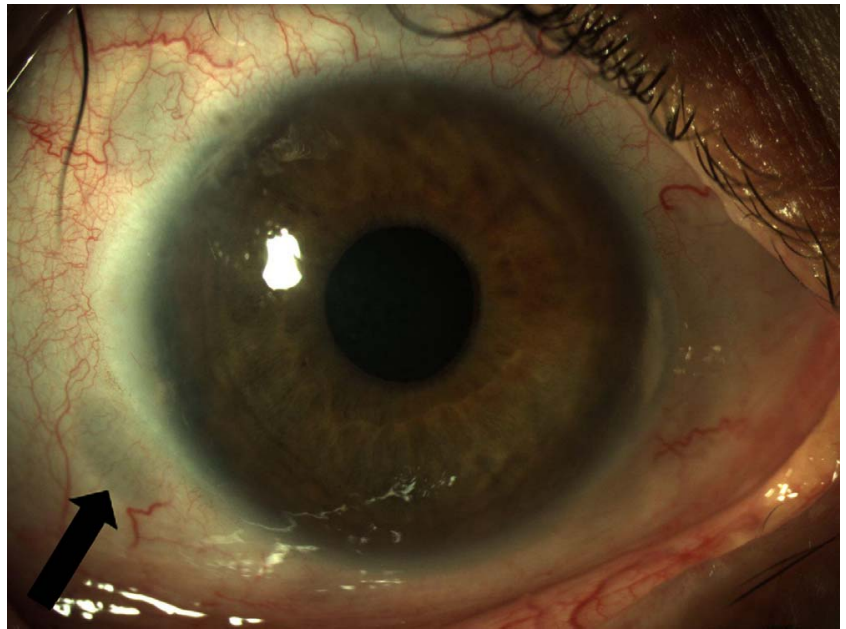

Figure 5. Image showing localized and nontransilluminable scleral thinning after ultrasound circular cyclo-coagulation (UC3) cyclophotocoagulation. Note that the regions appear to be well delineated, without any signs of conjunctival inflammation, induced corneal astigmatism, or scleral protrusion. Black arrow indicates area on 7o'clock meridian treated.

The tolerability of HIFU cyclocoagulation was good in the present study, as reported in the two previous studies, with no IOP spikes or major IOP increases during the early follow-up and over the long term no cases of severe hypotony or phthisis, which are some of the most serious adverse effects of the currently available cyclodestructive methods. Clinical examinations showed little or no signs of intraocular inflammation (Figs. 4,5 ) and visual acuity remained statistically unchanged in most of the patients.

\section{Conclusions}

In summary, the present multicenter study showed that ultrasonic coagulation of the ciliary body, using high-intensity focused ultrasound, is effective in decreasing IOP, particularly in patients with POAG. A substantial increase in ultrasound exposure time does not seem to increase the rate of responders and the global efficacy of the technique.

\section{Acknowledgments}

The authors thank Esther Blumen-Ohana and Pascale Hamard for their participation in the Clinical Trial.

Disclosure: P. Denis, EyeTechCare (C); F. Aptel, EyeTechCare (C); J.-F. Rouland, EyeTechCare (C); J.-P. Nordmann, EyeTechCare (C); Y. Lachkar, None; J.-P. Renard, EyeTechCare (C); E. Sellem, EyeTechCare (C); C. Baudouin, None; A. Bron, None

\section{References}

1. Kass MA, Heuer DK, Higginbotham EJ, et al. The Ocular Hypertension Treatment Study: a randomized trial determines that topical ocular hypotensive medication delays or prevents the onset of primary open-angle glaucoma. Arch Ophthalmol. 2002;120:701-713.
2. Heijl A, Leske MC, Bengtsson B, et al. Reduction of intraocular pressure and glaucoma progression: results from the Early Manifest Glaucoma Trial. Arch Ophthalmol. 2002;120:12681279 .

3. The effectiveness of intraocular pressure reduction in the treatment of normal-tension glaucoma. Collaborative NormalTension Glaucoma Study Group. Am J Ophthalmol. 1998;126: 498-505.

4. Vernon SA, Koppens JM, Menon GJ, et al. Diode laser cycloablation in adult glaucoma: long-term results of a standard protocol and review of current literature. Clin Experiment Ophthalmol. 2006;34:411-420.

5. Jennings BJ, Mathews DE. Complications of neodymium:YAG cyclophotocoagulation in the treatment of open-angle glaucoma. Optom Vis Sci. 1999;76:686-691.

6. Kirwan JF, Shah P, Khaw PT. Diode laser cyclophotocoagulation: role in the management of refractory pediatric glaucomas. Ophthalmology. 2002;109:316-323.

7. Walland MJ. Diode laser cyclophotocoagulation: longer term follow up of a standardized treatment protocol. Clin Experiment Ophthalmol. 2000;28:263-267.

8. Frezzotti P, Mittica V, Martone G, et al. Long-term followup of diode transscleral cyclophotocoagulation in the treatment of refractory glaucoma. Acta Ophthalmol. 2010;88:150-155.

9. Iliev ME, Gerber S. Long-term outcome of trans-scleral diode laser cyclophotocoagulation in refractory glaucoma. $\mathrm{Br} \mathrm{J}$ Ophthalmol. 2007;91:1631-1635.

10. Pucci V, Tappainer F, Borin S, et al. Long-term follow-up after transscleral diode laser photocoagulation in refractory glaucoma. Ophtalmologica. 2003;217:279-283.

11. Coleman D, Lizzi F, Driller J, et al. Therapeutic ultrasound in the treatment of glaucoma, II: clinical applications. Ophthalmology. 1985;92:347-352.

12. Valtot F, Kopel J, Haut J. Treatment of glaucoma with high intensity focused ultrasound. Intern Ophthalmol. 1989;13: 167-170.

13. Muratore R. A history of the Sonocare CST-100: the first FDAapproved HIFU device. In: Clement GT, McDannold NJ, Hynynen K, eds. 5th International Symposium on Therapeutic Ultrasound. Vol. CP829, AIP Conference Proceedings. Boston, MA: American Institute of Physics; 2005:508-512.

14. Burgess SE, Silverman RH, Coleman DJ, et al. Treatment of glaucoma with high intensity focused ultrasound. Ophthalmology. 1986;93:831-838.

15. Aptel F, Lafon C. Therapeutic applications of ultrasound in ophthalmology. Int J Hyperthermia. 2012;28:405-418.

16. Charrel T, Aptel F, Birer A, et al. Development of a miniaturized HIFU device for glaucoma treatment with conformal coagulation of the ciliary bodies. Ultrasound Med Biol. 2011;37:742754.

17. Aptel F, Charrel T, Lafon C, et al. Miniaturized high-intensity focused ultrasound device in patients with glaucoma: a clinical pilot study. Invest Ophthalmol Vis Sci. 2011;52:8747-8753.

18. Aptel F, Charrel T, Palazzi X, Chapelon JY, Denis P, Lafon C. Histologic effects of a new device for high-intensity focused ultrasound cyclocoagulation. Invest Ophthalmol Vis Sci. 2010; 51:5092-5098.

19. Aptel F, Dupuy C, Rouland JF. Treatment of refractory openangle glaucoma using ultrasonic circular cyclocoagulation: a prospective case series. Curr Med Res Opin. 2014;30:15991605. 